\title{
How to Design an Integration Platform for Interoperable
}

\section{EHR?}

\author{
Daniel Krsička ${ }^{1}$, Milan Šárek ${ }^{2}$ \\ ${ }^{1}$ First Faculty of Medicine, Charles University in Prague, Czech Republic \\ ${ }^{2}$ CESNET z.s.p.o., Prague, Czech Republic
}

\begin{abstract}
Background: Integration platform is a basic technical tool realizing an interoperable Electronic Health Record (EHR). Objectives: Our goal is an analysis of the integration platform functional structure and its relations to defined interoperability levels.

Methods: The existence possibility of a simple dependency between EHR use cases and integration platform technical functions will be tested on the models.
\end{abstract}

\section{Correspondence to:}

\section{Daniel Krsička}

First Faculty of Medicine, Charles University in Prague

Address: Kateřinská 32, 12108 Prague 2, CR

E-mail: dkrsicka@gmail.com
Results: The experiments will result into a proof of existence of this dependency and into a possibility to work with it.

Conclusions: The results will be discussed according to opportunity to generalize this method, to use it practically and develop further research in this domain.

\section{Keywords}

Interoperability, electronic health record, healthcare information system, integration platform, integration pattern

\section{EJBI 2012; 8(5):9-18}

recieved: August 15, 2012

accepted: October 15, 2012

published: November 22, 2012

\section{Introduction}

Massive penetration of the Healthcare Information Systems (HIS) and eHealth resources in general potentiate the significance of Electronic Health Record (EHR) interoperability as an ability of two or more subjects to achieve a common goal or mutually support each other to achieve the individual goals respectively (synergic effect). To describe this effect better, we can use the Metcalf's Law, postulated originally for telecommunication networks and Ethernet. This law introduces a network value quantity described as the number of all possible connections among subscribers (HIS in our case). So value of the whole interoperable EHR system should be dependent on the number of systems (HISs) integrated and asymptotically approximated by the quadratic polynomial of $n^{2}$.

Nevertheless it is becoming apparent [6] that the value of integrated HISs as a whole is not growing quadratic and that the Metcalf's Law is not applicable as a sufficient model. The reason is simple - Metcalf's law omits these parts of reality, essential for EHR interoperability analysis, primarily facts regarding EHR messages content and its usage in work (business) processes. The HIS integration is not a mere communication interconnection, so it is not about connection establishment only. It is necessary to pinpoint and follow many protocols enabling an information interchange for particular HIS components and layers. That implies the definition of interoperability level.

Table 1: Interoperability level definitions comparison.

\begin{tabular}{|c|c|}
\hline Levels after Bloebel & Levels after Gibbons \\
\hline \hline Process / Service & Process \\
\hline Semantic & Semantic \\
\hline Syntactic & Technical \\
\hline Structural & Technical \\
\hline Technological & Technical \\
\hline
\end{tabular}

We can use the existing definition after Gibbons 14 postulated in scope of HL7 EHR Interoperability Workgroup, defining 3 levels, or we can use the definition after Bloebel [1] setting up 5 levels of interoperability. Researching aforementioned resources we have defined relations among these 2 definitions, figured out in Table 1. For our purposes we will use the interoperability levels definition after Bloebel onwards, who demonstrates insufficiency of the traditional interoperability perception in technological degree and emphasizes the higher interoperability levels including the semantic. The classification 
after Gibbons is not suitable for our work due to our focus on logical integration platform design which is in Gibbon's definition plainly abstracted into just one technical interoperability level.

Our motivation is based on lessons learned about the technological interoperability insufficiency as a means of massive dissemination of interoperable EHR including all needed attributes defined e.g. in ISO/EN:13606 [10]. This statement is supported by the professional publications focusing mainly on EHR system content and semantics. Oneself, we have published the technological interoperability view inadequacy in $[15]$ and [16]. We have demonstrated that the higher interoperability levels cannot be assured by and based on accepted and broadly used classification into technical layers according to ISO/OSI model in ISO/IEC:7498 [17]. The process and partly the semantic interoperability has not any technical equivalent in ISO/OSI model, so these interoperability levels cannot be procured by technical resources only.

The present professional publications aiming interoperability are concentrating primarily on issues of EHR standardization, its structuring, content and usage by the end users including the semantic interoperability support in the form of data standard definitions, common vocabularies and ontologies. An EHR functional model is published in ISO/HL7:10781 [11] defining a basic set of EHR use cases. Unfortunately, the functional view research combining the EHR requirements with the technical realization of EHR integration platform in considerably underestimated in the professional society. Basic architectures of some national EHR projects, systems or efforts can be found. There are some groups like HSSP [8] engaged in EHR integration platform definitions, nevertheless a generally usable, comprehensive, logical design of the integration platform internal mechanisms as a functional composite of more than one HIS, aggregating the substantial functions centrally is not published yet.

The HIS semantic interoperability can be significantly supported by usage of EHR standards like HL7 [9] or DASTA [13] in the Czech environment. Each standard proceeds from its basic metamodel serving for deriving all the other parts of the standard. This metamodel also restricts the area and intent of standard application. This can be demonstrated on comparison between the standards HL7v3 and DASTAv3. HL7 is based on its Reference Information Model (RIM) establishing a basic "skeleton" for all the HL7 models as a relation among the subject, role, activity and object. Using this paradigm, all the relations of this type can be sufficiently described by the HL7v3 in the same way. On the other hand, the Czech national standard DASTAv3 bases its structure on information descriptive view only. It does not cover the interaction among various EHR roles, so it is good usable for data description, but unusable for the semantic expressions or managing work (business) processes. This difference has been well described and practically demonstrated by examples in [5].
As described further, to reach the highest interoperability level is not necessary and should not be an automatic goal for each HIS, because not each interoperable EHR system has to implement all the interoperability functions defined. The driving factor form the specific HIS use cases resulting in requirements on interoperability of particular level.

Our goal is to point to the importance of functional approach to the EHR communication, to verify possibilities of present semantic interoperability knowledge utilization for an integration platform design methods simplifying and formalization.

\subsection{Integration Platform}

The integration platform is a basic technical means for integration of information systems, the HISs inclusive. It consists of hardware and software components and also data models, structures processing rules and security mechanisms. For our purposes we will focus on the software part of integration platform logical structure only. It is composed of a logical functions basic set, cooperatively realizing the EHR messages transport and processing. Further we will define the integration platform using these functions and their aggregations in relations to the particular interoperability levels.

\subsection{Integration Pattern}

Integration patterns are partial functional concepts, from whose realization the integration platform consists of. Each integration pattern [7] is a generalization of a verified method (best practice) in the area of information system integration. It is a special case of design pattern [18, typically defined by an unique syntactical graphical model and informal semantic description describing the case of pattern usage. For our work we have used probably the most comprehensive set of integration patterns published by Hohpe and Gregor [7]. The alphabetical order is depicted in the Figure 1 .

Each integration pattern solves a particular typical situation in data communication and processing through the integration platform. Particular EHR use cases mark off each other and each use case or even each EHR message transported among HISs can be processed in a different way, so different integration platform components can be used, thus different integration patterns and their combinations apply.

Our goal is to structure the mentioned ambiguity and define rules applicable in early EHR implementation project phases and simplifying the logical design significantly. This logical design has to be pure platform (technological) independent. The facilitation lies in the target interoperability level definition belonging to the specific organization (defined by its EHR use cases) and the proposal of basic integration platform logical structure (expressed in the sets of integration patterns to implement). 


\begin{tabular}{|c|c|c|c|c|}
\hline Aggregator & $\begin{array}{c}\text { Canonical Data } \\
\text { Model }\end{array}$ & $\begin{array}{l}\text { Channel } \\
\text { Adapter }\end{array}$ & $\begin{array}{l}\text { Channel } \\
\text { Purger }\end{array}$ & Claim Check \\
\hline $\begin{array}{c}\text { Command } \\
\text { Messaqe }\end{array}$ & $\begin{array}{l}\text { Competing } \\
\text { Consumers } \\
\end{array}$ & $\begin{array}{c}\text { Composed } \\
\text { Messaqe }\end{array}$ & $\begin{array}{l}\text { Content } \\
\text { Enricher }\end{array}$ & Content Filter \\
\hline $\begin{array}{c}\text { Content-based } \\
\text { Router }\end{array}$ & Control Bus & $\begin{array}{c}\text { Correlation } \\
\text { Identifier }\end{array}$ & $\begin{array}{l}\text { Datatype } \\
\text { Channel }\end{array}$ & $\begin{array}{c}\text { Dead Letter } \\
\text { Channel }\end{array}$ \\
\hline Detour & $\begin{array}{c}\text { Document } \\
\text { Messaqe }\end{array}$ & $\begin{array}{c}\text { Durable } \\
\text { Subscriber }\end{array}$ & $\begin{array}{c}\text { Dynamic } \\
\text { Router } \\
\end{array}$ & $\begin{array}{l}\text { Envelope } \\
\text { Wrapper }\end{array}$ \\
\hline Event Message & $\begin{array}{l}\text { Event-driven } \\
\text { Consumer }\end{array}$ & File Transfer & $\begin{array}{c}\text { Format } \\
\text { Indicator }\end{array}$ & $\begin{array}{c}\text { Guaranteed } \\
\text { Delivery }\end{array}$ \\
\hline $\begin{array}{c}\text { Idempotent } \\
\text { Receiver }\end{array}$ & $\begin{array}{l}\text { Invalid } \\
\text { Messaqe }\end{array}$ & $\begin{array}{c}\text { Message } \\
\text { Broker }\end{array}$ & Message Bus & $\begin{array}{l}\text { Message } \\
\text { Channel }\end{array}$ \\
\hline $\begin{array}{c}\text { Message } \\
\text { Dispatcher }\end{array}$ & $\begin{array}{l}\text { Message } \\
\text { Endpoint }\end{array}$ & $\begin{array}{l}\text { Message } \\
\text { Expiration }\end{array}$ & Message Filter & $\begin{array}{c}\text { Message } \\
\text { History } \\
\end{array}$ \\
\hline $\begin{array}{c}\text { Message } \\
\text { Router }\end{array}$ & $\begin{array}{l}\text { Message } \\
\text { Sequence }\end{array}$ & Message Store & $\begin{array}{l}\text { Message } \\
\text { Translator }\end{array}$ & Message \\
\hline $\begin{array}{c}\text { Messaging } \\
\text { Bridqe } \\
\end{array}$ & $\begin{array}{c}\text { Messaging } \\
\text { Gateway }\end{array}$ & $\begin{array}{c}\text { Messaging } \\
\text { Mapper } \\
\end{array}$ & Messaging & Normalizer \\
\hline $\begin{array}{l}\text { Pipes and } \\
\text { Filters } \\
\end{array}$ & $\begin{array}{c}\text { Point-to-Point } \\
\text { Channel }\end{array}$ & $\begin{array}{c}\text { Polling } \\
\text { Consumer }\end{array}$ & $\begin{array}{l}\text { Process } \\
\text { Manaqer }\end{array}$ & P/S Channel \\
\hline Recipient List & $\begin{array}{c}\text { Remote } \\
\text { Invocation }\end{array}$ & $\begin{array}{c}\text { Request / } \\
\text { Reply }\end{array}$ & Resequencer & Return Address \\
\hline Routing Slip & $\begin{array}{l}\text { Scatter / } \\
\text { Gather }\end{array}$ & $\begin{array}{l}\text { Selective } \\
\text { Consumer }\end{array}$ & $\begin{array}{c}\text { Service } \\
\text { Activator }\end{array}$ & $\begin{array}{c}\text { Shared } \\
\text { Database } \\
\end{array}$ \\
\hline Smart Proxy & Splitter & Test Message & $\begin{array}{c}\text { Transactional } \\
\text { Client }\end{array}$ & Wire Tap \\
\hline
\end{tabular}

Figure 1: Alphabetical list of the integration patterns - the atomic EHR integration platform funcionalities.

Of course it will be a generic basis only which should be analyzed and customized in deeper detail during the EHR implementation project. Anyway a data analysis should be established on the advanced standards like HL7 and the project should follow a wide accepted methods like Rational Unified Process (RUP) [20].

We expect that some integration patters are already whole or partially included in existing standards like IHE [19 or that existing standards are tight coupled to them. More information can be found in the section Discussion of this article.

\subsection{EHR Use Cases}

The use case forms the usage specification of particular HIS function by an external role (outside the system) like user, other system etc. The typical EHR use cases can be found in 11, 6 6 or in [19. The test cases of EHR use cases can be found in section Experiments of this article.

\section{Goals and Hypotheses}

We focus on the functional view analysis of EHR integration platform as a technical means of interoperable EHR realization. EHR integration between 2 HISs is supported by an integration platform. Its structure and behaviour has to include all the functions necessary for reaching the target EHR interoperability level. Therefore we need to find dependencies among EHR requirements, interoperability level requirements and structure of the integration

We would like to elaborate a formal method supporting the EHR use case analysis which would simplify and speed up an integration platform design. This way an interoperable EHR implementation would be supported. Aforementioned method benefits lie in analysis and design acceleration, implementation shortening, support of early prototype creation and anticipated decreasing the number of change request, so in reduction of total solution costs. 


\section{Hypothesis}

Let us suppose that there is a mapping, assigning for each EHR use case a set of integration patterns. These patterns ensure the EHR integration platform functionalities required for the use case realization and will correspond to the necessary interoperability level. We propose that by a sequential aggregation of these mappings it will be possible to prepare a basic functional structure for the whole EHR integration platform required for particular set of EHR use cases (business requirements).

Let us structure the functionality sets of EHR integration platform according to the interoperability level needed and try to find a mapping from the set of use cases to this structure. It should result into a definition of assignment from set of EHR use cases into a necessary interoperability level.

The benefit is a software analysis simplification and EHR integration platform design optimization.

\section{Methods}

\section{Interoperability Related Classification of Integration Patterns}

To enable an assignment of each integration pattern to the typical HIS interoperability level, it has been inevitable to establish a hierarchical model of integration patterns. This hierarchy follows the interoperability levels and also the typical structure of integration platform, i.e. transport and processing parts, but without generally accessible business services, which can be established with data semantic usage only. This structure introduces a basic technical means for EHR integration among systems (HISs interoperable integration). Descriptions of individual integration platform layers follow:

- Access Layer: forms a place, where all the integrated systems connect to, to establish a suitable communication. It contains algorithms and structures enabling technical resources compatibility. From the ISO/OSI perspective it is a solution on layers 1 to 5.

- Transport Layer: ensures a basic user data transmission up to the ISO/OSI layer 6. Data is encapsulated into messages. During the analysis, it is necessary to define the technical metadata determining communication endpoints and data structures. Transport layer takes care about all the transmission mechanisms including failover, high-availability, reliability or idempotence.

- Transformation and Routing Layer: manipulates with data transmitted within the meaning of format and structure change on the ISO/OSI layer 7. There is a necessary condition of existence and compliance with common registers, vocabularies and rules. The layer also routes the messages, their parts or aggregations to the right recipients.

- Semantic Layer: Works with the meaning of transmitted information. Components of this layer has to be able to ensure communication among mutually heterogeneous business (or information) domains within the meaning of Generic Component Model [3]. The semantic layer algorithms focus on the data meaning, nor on the data structure or information syntax. In contrast to the well known accord [9], we suppose that this layer has not an equivalent layer in the ISO/OSI model, because this does not solve the data transport, but presentation and sense only.

- Business Processes Layer: concentrates on processes executed by given roles. These processes can have a known structure or be dynamic and to progress according to the actual system state, environmental (contextual) information and to the data processed by the layer. It also includes processes solving a feedback-based process / system optimization. It has not an equivalent in ISO/OSI model.

Above mentioned integration platform structure enables an assignment of corresponding interoperability levels after Bloebel et alli 1] 2] 4. Here we are looking for and testing a relation (dependency) between EHR / HIS interoperability levels and integration platform layers.

By a combination of interoperability levels and semantics of particular integration patterns, we obtained an integration patterns set structuring into the 5 subsets. For more information see the Figure 2. We propose that the EHR use cases majority will be resolvable by some of these patterns of particular subsets. But to do this, we have to evaluate the EHR use cases and assign a necessary interoperability level to each use case according to specific method. Thus we need a classification or evaluation system for the EHR use cases. This system is suggested in the next chapter.

\section{EHR Use Cases Classification}

To prepare an EHR use case structuring it is appropriate to define them the classification criterions with following features:

- applicable universally to any EHR use case,

- with trivial semantics excluding misunderstanding and facilitating the utilization,

- moderate number of possible values.

Inspired by the HL7v3 RIM [9] and the law of 5W [21] we have proposed following classification criterions for the EHR use cases: 


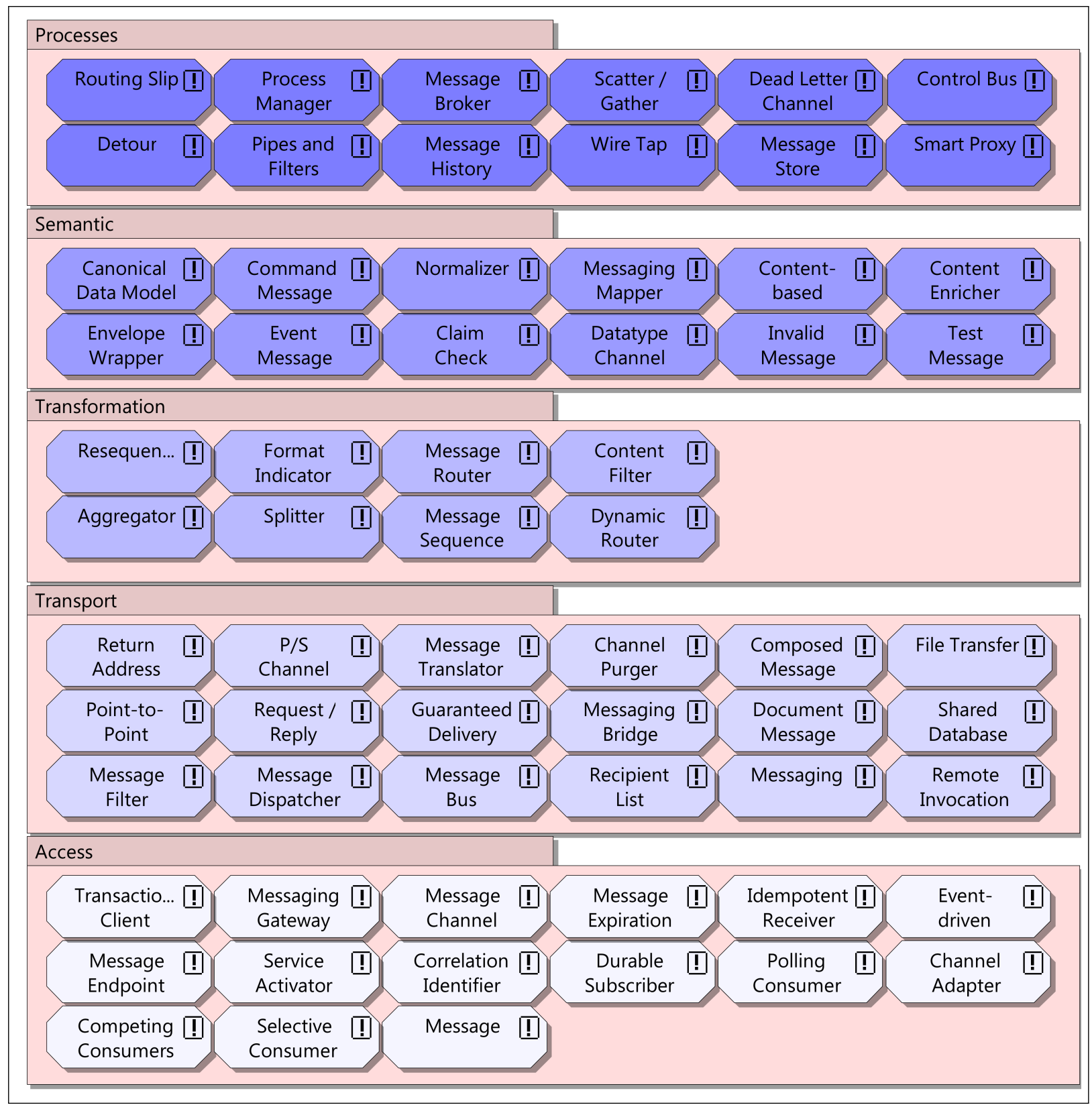

Figure 2: Integration patterns divided into the groups each supporting a particular level of interoperability.

- Space - reflecting the perspective given by questions: "Where the information communication takes place? How distant the points of presence are?"

- Time - reflecting the perspective given by questions: "When the communication takes place? How fast and often it runs?"

- Subject - reflecting the perspective given by questions: "Who is communicating? What is his skills?

- Object - reflecting the perspective given by questions: "What is communicated? Why the communication runs?"

For our experimental purposes we draft weighted values of these classification criterions:

\section{The Dimension of Space}

Considering the interoperability perspective, the physical distance of communicating roles is not so important in comparison with the logical distance emerging from the mutual conversance of communicating roles. It can be distinguished in 2 groups. The first one forms persons, i. e. there is difference between information sharing e. g. the physician and nurse in one hospital department or whether communicate a GP with a specialized detached laboratory. Due to we are modelling with point to optimize logical design of technological components, we omit the cultural and social specifics. The second group incorporates the organizations and we can scale, as in the first group, from private praxis, particular hospital de- 
partments, clinics, hospitals to insurance companies and national healthcare-related institutions.

For EHR use case ration we will apply 1 from 3 following values possible and the corresponding score.

- Communication in a work team (0 points) - The communicating know each other in person. Communication runs in real time and brings a lower formalization level.

- Communication in an organization (1 point) - The particular communicating are motivated by the same goals and common working methods in outline.

- Communication between organizations (2 points) Strictly formal communication way with necessity to establish a contract for all the services provided or consumed between organizations.

\section{The Dimension of Time}

The time dimension impacts the EHR integration mostly in the requirement specification (business processes or use cases) and in the technical realization. On the other hand, the application of data standards is less affected. The necessary interoperability level is not influenced by the time dimension directly, but it is a suitable additional information to the use case specification and it will be used for the analysis and particular implementation design. It is important to see that it characterizes the data access frequency and so amount of the formalization required (data not red or changed become obsolete and unreadable). We propose the following weights and score.

- Real time communication (0 points) - Information interchanged immediately after creation and often also immediately utilized. Typical examples are daily records, statim indications etc.

- Daily communication (1 point) - Information interchange once or more times a day, Mostly it is regarding to the primary (business) processes like a care provision.

- Monthly communication (2 points) - Communication of often aggregated data. The indication arises from lower use case criticality or from necessity to process data in the batch transactional way (e.g. reporting for payments or perhaps data mining for statistical studies with need to lock a large data set for a while to ensure consistency).

\section{The Dimension of Subject}

For our experiment a small set of role is enough. For the comprehensive set definition a concept from ISO/TS:22600 [16 can be used. For consideration of necessary interoperability level it is much more important to evaluate the differences among communicating roles regarding specialization and education of communications. For definition of the subject dimension meaning we use the Generic Component Model 3, its Domain Perspective dimension respectively.

- Roles with the same knowledge (0 points) - Roles in the communication have approximately the same education and specialization. They work in the same or similar processes, activities and their aspects. They understand the same terminology and paradigms. E.g. physicians in the same department

- Roles with a similar knowledge (1 point) - Communicating roles works in the same discipline (domain), but they do not have the same education and knowledge. In this domain they perform different activities. They understand a certain common language and terminology, but each of them maintain its own specializations. Examples can be physician and nurse, physicians of different specializations, scientist in primary research and clinical doctor etc.

- Roles with completely different knowledge (2 points) - The roles have completely different education and knowledge. They a priori do not understand the opposite role principles and means of expression. Confronted with a particular problem or question they focus on different aspects and apply different approaches to the solution. Typical comparison can be physician and patient, administrative worker and manager, ...

\section{The Dimension of Object}

At first sight, the communication object classification is quite complex due to its diversity and set cardinality. Nevertheless with regards to the classification model intent an analysis of particular attributes is enough and so we do not need to know the complete messages content. Our goal is to design a logical structure of technical resources (components), not their content like rules, algorithms, registers or vocabularies. So we focus on syntax and semantics expression in the transferred messages. With regard to possible interpretation after [3] we define the following criterion values:

- Usage of syntax (0 points) - The information shared is written in a formalized way. Data is readable by machines in platform independent way, the data structures are defined with use of EDI, XSD, ... and also shared registers.

- Usage of semantics (1 point) - Includes the Syntactic group attributes and also use metadata defining the meaning and sense (for the end user or for processing engines) of transmitted information. This enables a sharing among different roles thanks the information unambiguity. 
- Usage for deterministic action (2 points) - The transmitted information is structurally and semantically deterministic enough to execute and automatic processing in HIS or to propose a working method /process for a role a priori unskilled in the domain / profession. For example the advanced systems for decision support or automatic business process management such as optimization and planning processes.

In this article we disregard other partial classification, namely the questions of technological data records and their structuring. These attributes influences the data modelling which is out of scope of this article.

\section{EHR Use Case Classification and the Interoperability Levels}

The basic classification challenge in the proposed method is a derivation of target interoperability level from the values of aforementioned classification criterions. Each EHR use case can get from 0 to 8 points in total (4 criterions, 0 - 2 points in each criterion). After more detailed consideration we conclude that the summation is not the primary but much more important is the combination of criterion values. For assessment we specify rules in Table 2.

Table 2: Classification criterion values evaluation.

\begin{tabular}{|c|c|c|}
\hline 1 earned & 2 earned & Target Interoperability Level \\
\hline- & $\geq 2$ & Process \\
\hline- & $\geq 1$ & Semantic \\
\hline$\geq 1$ & - & Syntactic \\
\hline 0 & 0 & Structural, Technical \\
\hline
\end{tabular}

\section{Aggregation Results in EHR Integration Platform Design}

In the following experiments we are going to classify each model EHR use cases according to the criterions. We will get a set of pairs [use case; interoperability level]. Based on the highest interoperability level required in this set and with regard to the distribution of their relative frequencies we suppose to design an initial EHR integration platform layers. These layers are defined by sets of integration patterns as the basic functionalities of each layer. Analysis in a specific implementation project should focus just on these layers. From the relative frequencies distribution we can expect the majority of analytical work in the project. Let us show on 2 small examples

\section{Model Situation Nr. 1:}

A small purpose-built application for one clinical department, 25 use cases in total. Distribution of interoperability levels required is in Table 3 .
Table 3: Interoperability level required by use cases in example Nr. 1.

\begin{tabular}{|c|c|}
\hline Interoperability level & Number of cases \\
\hline \hline Technical & 24 \\
\hline Structural & 20 \\
\hline Syntactic & 18 \\
\hline Semantic & 2 \\
\hline Process & 0 \\
\hline
\end{tabular}

Conclusion: The initial integration platform design has to be focuses on technological compatibility, transport protocols and messages format standardization.

\section{Model Situation Nr. 2:}

2 HISs integration between 2 independent hospitals, 250 use cases in total. Distribution of interoperability levels required is in Table 4

Table 4: Interoperability level required by use cases in example Nr. 2.

\begin{tabular}{|c|c|}
\hline Interoperability level & Number of cases \\
\hline \hline Technical & 250 \\
\hline Structural & 230 \\
\hline Syntactic & 200 \\
\hline Semantic & 180 \\
\hline Process & 40 \\
\hline
\end{tabular}

Conclusion: The initial integration platform design has to encompass the support of access, transport, transformation and routing of data based on technical and also user metadata. Processes (workflow) are defined within the services between hospitals and a request for orchestration emerges. This can be realized by specializes process interoperability integration patterns and components (broadly by an orchestration engine).

\section{Experiments - Model EHR Use Cases and Interoperability}

We have applied the aforementioned method on 6 following model EHR use cases. Each use case has been defined by its initial (business) description. Usually the description is supplemented during the analysis phase with the customer (e.g. physicians). In our experiments we have used our own information and knowledge for the simulation.

The overall use case semantics has been evaluated under given classification criterions and we obtained the required combinations of weighted values. Based on these combinations we set the required interoperability level for each EHR use case.

Aggregating all the experiments together we gained the relative distribution of interoperability level frequencies as a basis for an initial EHR integration platform design. 


\subsection{Experiment Nr. 1}

Use case description: Management of daily records in one clinical department.

Analysis: The roles work in a compact team, the coworker know each other and all belongs to one professional domain.

Classification: can be found in Table 5

Table 5: Use cases evaluation in experiment Nr. 1.

\begin{tabular}{|c|c|}
\hline Criterion & Valuee / Score \\
\hline \hline Space & in team / 0 \\
\hline Time & real time / 0 \\
\hline Subject & similar / 1 \\
\hline Object & syntactic / 0 \\
\hline
\end{tabular}

Conclusion: Interoperability level required for use case Nr. 1 is: Syntactic.

\subsection{Experiment Nr. 2}

Use case description: Access to the patients radiological data for other physicians.

Analysis: The co-workers do not need to know each other and their specialization can (and probably will) differ, even if we suppose a quite good knowledge and experience with reading the results from visualization methods (here RTG).

Classification: can be found in Table 6

Table 6: Use cases evaluation in experiment Nr. 2.

\begin{tabular}{|c|c|}
\hline Criterion & Value / Score \\
\hline \hline Space & in organization $/ 1$ \\
\hline Time & real time / 0 \\
\hline Subject & similar $/ 1$ \\
\hline Object & semantic / 1 \\
\hline
\end{tabular}

Conclusion: Interoperability level required for use case Nr. 2 is: Syntactic.

\subsection{Experiment Nr. 3}

Use case description: Patient's laboratory test results access for a GP, processed by an external testing laboratory.

Analysis: Cooperating roles do not know each other. There is no need for real time communication. The specialization and knowledge can differ but the most common tests have to be able to read all the physicians. We do not consider the special laboratory tests (like CVS, cancer marks, detailed haematology or immunology) which are not commonly indicated by GPs. The functionality can be offered as a service so the contract definition is necessary (SLA - Service Level Agreement).

Classification: can be found in Table 7 .
Table 7: Use cases evaluation in experiment Nr. 3.

\begin{tabular}{|c|c|}
\hline Criterion & Value / Score \\
\hline \hline Space & between orgs. / 2 \\
\hline Time & daily / 1 \\
\hline Subject & similar / 1 \\
\hline Object & semantics / 1 \\
\hline
\end{tabular}

Conclusion: Interoperability level required for use case Nr. 3 is: Semantic.

\subsection{Experiment Nr. 4}

Use case description: Access to the anonymized patient data in an university hospital from an university research centre for the purpose of a statistical longitudinal study.

Analysis: It is necessary to define not only content and semantics of the data but also the way and purpose of its processing. We have to respect the regulatory law and also must not omit some information relevant for the study (false positive/negative results risk).

Classification: can be found in Table 8 .

Table 8: Use cases evaluation in experiment Nr. 4.

\begin{tabular}{|c|c|}
\hline Criterion & Value / Score \\
\hline \hline Space & in organization / 1 \\
\hline Time & monthly / 2 \\
\hline Subject & similar / 1 \\
\hline Object & deterministic action / 2 \\
\hline
\end{tabular}

Conclusion: Interoperability level required for use case Nr. 4 is: Process.

\subsection{Experiment Nr. 5}

Use case description: Reporting of provided healthcare from the provider to the payer.

Analysis: A periodical rigid communication in the form of a service provided and consumed among organizations (more service consumers / healthcare providers). The contract (SLA) definition is absolutely inevitable.

Classification: can be found in Table 9.

Table 9: Use cases evaluation in experiment Nr. 5.

\begin{tabular}{|c|c|}
\hline Criterion & Value / Score \\
\hline \hline Space & between organizations / 2 \\
\hline Time & monthly / 2 \\
\hline Subject & different / 2 \\
\hline Object & semantic / 1 \\
\hline
\end{tabular}

Conclusion: Interoperability level required for use case Nr. 5 is: Process. 


\subsection{Experiment Nr. 6}

Use case description: On-line access for the patient to his/her EHR.

Analysis: Ad hoc access which realization request emerges from the valid Czech law. The patient (user) stays out of the organization, its motivation, knowledge and experience is completely different in comparison with healthcare professionals. The accessible EHR must include also additional information enabling the patient's understanding.

Classification: can be found in Table 10 .

Table 10: Use cases evaluation in experiment Nr. 6.

\begin{tabular}{|c|c|}
\hline Criterion & Value / Score \\
\hline \hline Space & between organizations / 2 \\
\hline Time & real time / 0 \\
\hline Subject & different $/ 2$ \\
\hline Object & semantic $/ 1$ \\
\hline
\end{tabular}

Conclusion: Interoperability level required for use case Nr. 6 is: Semantics.

\section{Results}

Based on the knowledge about particular interoperability levels and with use of classification rules mentioned above we have evaluated a required interoperability level in each model EHR use case. Thus we have demonstrated that a mapping required in our hypothesis really exists and that for the level definition we can use quite simple classification criterions, understandable also for persons not skilled in computer science. We have demonstrated that required mapping can be found for aforementioned the EHR use cases, because of classification according to generic criterions.

It is clear form experiment's results Nr. $1-6$ how the model integration platform design looks like. It is determined by the highest interoperability level found in the use cases and by the relative distributions of levels found. Let us summarize this data in Table 11 .

Table 11: Aggregation of experiments results.

\begin{tabular}{|c|c|}
\hline Required Interoperability Level & Total incidences \\
\hline Process & 2 \\
\hline Semantic & 4 \\
\hline Syntactic & 6 \\
\hline Structural & 6 \\
\hline Technical & 6 \\
\hline
\end{tabular}

Looking on the table it is evident that this model situation has to base the initial integration platform design on common access, transport and transformation \& routing layer as an inevitable basis. Also an essential functional support for semantic interoperability is necessary.
The dedicated process engine realizing the integration patterns from the highest level should be considered, because its commonly a little bit expensive, so it could not be justified just for 2 use cases. But in the real project, if the Process interoperability forms more than $30 \%$ of total requirements, a standalone orchestration engine is absolutely needed.

\section{Discussion}

The classification rules for EHR use cases mentioned in this article can be apparently applied on any EHR use case and so it should be possible to evaluate any of them. The understanding of these rules is quite simple so the use cases can be evaluated also by a person without a specialized training in computer science and software engineering (physician, manager ...). This way a mapping between different GCM domains [3] is enabled in the integration platform development process. The definition of target interoperability results from the method stated in this article.

The method implication lies in the possibility to structured view to the often heterogeneous set of (business) requirements. For optimal method set up it is necessary to execute more experiments and tests on model and also real EHR use cases. It has to be tested whether the method can really simplify the analysis project phase and enable the development of an early integration platform prototype. The benefit of early prototype is the possibility to test soon after the requirement specification, to decrease the number of change requests, to speed up the project and to lower the costs in total.

According to our present research, it seems that some of presented integration patterns forming the range of values of our mapping already exist or are partly included in existing standards like the IHE profiles [19. These standards define the specific EHR use cases with some realization specifications inclusive. In the further research it will be appropriate to focus also on relations among these standards and logical functionality view represented by the integration patterns and their classification mentioned here.

\section{Conclusion}

With regard to the cost cutting need and the EHR implementation projects acceleration we have defined a supporting method for the EHR use case analysis. By application of this method we have obtained an information set for a logical, platform independent design of an EHR integration platform. The testing on model situations was successful and we are motivated for the further experiments including the real use cases in the healthcare provider environment. We expect that these tests together with further method advancement will be executed in the environment of Krajska zdravotni, the major healthcare provider in district of Ustecky kraj, incorporating 5 hos- 
pitals and cooperating on science and research. A part of this research should be also a comprehensive analysis of relations among various integration patterns and existing IHE profiles.

Building up the dedicated integration platforms is a natural evolutionary result of ICT penetration not only into the healthcare and its related to quadratic growth of communications among HISs. Crossing a particular limit complexity indicated a need to formalize these communications in objective and also functional manner. So we expect the further development not only in the field of data standards but also in the functional perspective of healthcare integration platforms and EHR.

\section{Acknowledgements}

The paper has been supported by the SVV-2012-264 513 project of Charles University in Prague.

\section{References}

[1] Bloebel, B. Architectural Approach to eHealth for Enabling Paradigm Changes in Health. Methods of Information in Medicine. 2010, 49, s.123-134.

[2] Bloebel, B., Gonzalez, C., Oemig, F., Lopez, D., Nykanen, P., Ruotsalainen, P. The Role of Architecture and Ontology for Interoperability. Stud Health Technol Inform. 2010, 155, s.33-39.

[3] Bloebel, B., Oemig, F. What Is Needed to Finally Achieve Semantic Interoperability? In: Doessel, O., Schlegel, W. C. (Edrs.) IFMBE Proceedings 25/XII. 2012, p. 411-415

[4] Bloebel, B., Oemig, F., Gonzales, C., Lopez, D.: What is Missing in Health Informatics. Medical and care compunetics, 2010, 156, s.3-12.

[5] Nagy, M., HanzlicekA, P., Preckova, P., Riha, A., Dioszegi M., Seidl, L., Zvarova, J. Semantic Interoperability in Czech Healthcare Environment Supported by HL7 Version 3. Methods of information in medicine. 2010, 49, s.186-195.

[6] Benson, T. Principles of health interoperability HL7 and SNOMED. New York: Springer, 2012, ISBN 978-144-7128-007.

[7] Hohpe, G. Enterprise integration patterns: designing, building, and deploying messaging solutions. Boston: AddisonWesley, 2004, 683 s. ISBN 03-212-0068-3.
[8] Healthcare Services Specification Project:HSSP [online]. 2012 [cit. 2012-06-19]. Via: http://hssp.wikispaces.com

[9] Health Level Seven International:HL7 [online]. 2012 [cit. 201206-19]. Via: http://www.hl7.org

[10] ISO/EN 13606 Health informatics - Electronic health record communication. Geneva, Switzerland: International Organization for Standardization, 2008-2010.

[11] ISO/HL7 10781:2009 Electronic Health Record-System Functional Model, Release 1. Geneva, Switzerland: International Organization for Standardization, 2006-2009.

[12] ISO/TS 22600 Health informatics - Privilege management and access control. Geneva, Switzerland: International Organization for Standardization, 2006-2009.

[13] Datovy standard Ministerstva zdravotnictvy CR:DASTA [online]. 2012 [cit. 2012-06-19]. Via: http://dastacr.cz

[14] Gibbons, P. Coming to Terms: White Paper on Interoperability. In: HL7 [online]. 2007 [cit. 2012-08-14]. Via: http://www.hl7.org/documentcenter/public/wg/ehr /ComingtoTerms2007-03-22.zip

[15] Krsicka, D. Sarek, M. Automatizace vyuziti blokovych reseni pro vyvoj architektur IS. In: MEDSOFT 2012. Praha: Dum techniky CSVTS, 2012, s. 168-179. ISSN 1803-8115.

[16] Krsicka, D. Sarek, M. Integracni vzory a jejich automaticke vyhodnocovani. In: Medsoft 2011. Praha: Creative Connections, 2011, s. 146-149. ISSN 1803-8115.

[17] ISO/IEC 7498-1:1994. Information technology - Open Systems Interconnection: Basic Reference Model: The Basic Model. Geneva, Switzerland: International Organization for Standardization, 1997

[18] Fowler, M. Patterns of enterprise application architecture. Boston: Addison-Wesley, c2003, xxiv, 533 p. ISBN 03-211$2742-0$.

[19] Integrating the Healthcare Enterprise:IHE [online]. 2012 [cit. 2012-06-19]. Via: http://www.ihe.net

[20] Jacobson, I., Fowler, M., Rumbaugh J. Unified software development process. Boston: Addison-Wesley, 1999, 463 s. ISBN 02-015-7169-2

[21] Five Ws. In: Wikipedia: the free encyclopedia [online]. San Francisco (CA): Wikimedia Foundation, 2001- [cit. 2012-0815]. Via: http://en.wikipedia.org/wiki/Five_Ws 